\title{
The Clinical and Radiological Outcomes of Minimally Invasive Transforaminal Lumbar Interbody Single Level Fusion
}

\author{
Moon-Chan Kim¹, Hung-Tae Chung ${ }^{1}$, Dong-Jun Kim¹, Sang-Hyuk Kim², Sang-Ho Jeon² \\ 'Department of Orthopaedic Surgery, Busan Bumin Hospital, Busan, Korea \\ ${ }^{2}$ Department of Neurosurgery, Busan Bumin Hospital, Busan, Korea
}

Study Design: This is a retrospective study that was done according to clinical and radiological evaluation.

Purpose: We analyzed the clinical and radiological outcomes of minimally invasive transforaminal lumbar interbody single level fusion.

Overview of Literature: Minimally invasive transforaminal lumbar interbody fusion is effective surgical method for treating degenerative lumbar disease.

Methods: The study was conducted on 56 patients who were available for longer than 2 years (range, 24 to 45 months) follow-up after undergoing minimally invasive transforminal lumbar interbody single level fusion. Clinical evaluation was performed by the analysis of the visual analogue scale (VAS) score and the Oswestry Disability Index (ODI) and the Kirkaldy-Willis score. For the radiological evaluation, the disc space height, the segmental lumbar lordotic angle and the whole lumbar lordotic angle were analyzed. At the final follow-up after operation, the fusion rate was analyzed according to Bridwell's anterior fusion grade.

Results: For the evaluation of clinical outcomes, the VAS score was reduced from an average of 6.7 prior to surgery to an average of 1.8 at the final follow-up. The ODI was decreased from an average of 36.5 prior to surgery to an average of 12.8 at the final follow-up. In regard to the clinical outcomes evaluated by the Kirkaldy-Willis score, better than good results were obtained in 52 cases (92.9\%). For the radiological evaluation, the disc space height ( $p=0.002$ ), and the whole lumbar lordotic angle ( $p=0.001$ ) were increased at the final follow-up. At the final follow-up, regarding the interbody fusion, radiological union was obtained in 54 cases (95.4\%).

Conclusions: We think that if surgeons become familiar with the surgical techniques, this is a useful method for minimally invasive spinal surgery.

Key Words: Minimally invasive, Transformainal, Lumbar interbody, Single level fusion

\section{Introduction}

Postlateral fusion using pedicle screws or posterior lumbar interbody fusion has traditionally been frequently performed for treating back pain and radiating pain caused by spinal instability, the degenerative process of the lumbar spine and the degenerative changes of the disc. Nonetheless, these procedures require the dissection of muscles and soft tissues in a wide area during surgery, which may increase the postsurgical back pain and lengthen the recovery period [1]. In addition, these methods may increase the volume of blood loss and damage nerves due to excessive neural retraction [2]. To compensate for such shortcomings, mini-

Received Jun 28, 2010; 1st Revised Aug 27, 2010; 2nd Revised Sep 27, 2010; 3rd Revised Oct 9, 2010; Accepted Oct 11, 2010

Corresponding author: Moon-Chan Kim, MD

Department of Orthopaedic Surgery, Busan Bumin Hospital, 380-4 Deokcheon 1-dong, Buk-gu, Busan 616-819, Korea

Tel: +82-51-330-3000, Fax: +82-51-337-5041, E-mail: moon_chan@hanmail.net 
mally invasive transforminal lumbar interbody fusion using a tubular retractor (METRx tube, Medtronic Sofamer Danek, Memphis, TN, USA) has recently been widely performed $[3,4]$. In this study, we assessed clinical and radiological results and the effectiveness of performing minimally invasive transforaminal lumbar interbody fusion using a tubular retractor in patients who were followed up for more than 2 years.

\section{Materials and Methods}

\section{Materials}

The study was conducted on 56 patients who underwent minimally invasive transforaminal lumbar interbody single level fusion from May 2006 to October 2007 and who underwent more than 2 years follow-up. The mean age of the patients was 58.7 years (range, 29 to 74 years) and the average follow-up period was 32.4 months (range, 24 to 45 months). As for the underlying diseases, there were 5, 22, 18 , and 11 cases of recurred disc herniation, degenerative spondylolisthesis, isthmic spondylolisthesis and foraminal stenosis, respectively. The patients who underwent fusion on more than two segments and the patients with spondylolisthesis higher than Meyerding grade II were excluded from the study.

\section{Surgical methods}

With the patient in the prone position and under general anesthesia, a skin incision $2-3 \mathrm{~cm}$ in length was made in the area $2.5-3.0 \mathrm{~cm}$ away from the spinous process. The smallest dilator was placed on the joint between the multifidus muscle and the longus colli muscle, the dilator was continuously advanced and the Metrix tube (METRx tube, Medtronic Sofamer Danek) of the appropriate length was inserted medially. After the removal of the soft tissues within the tube, the adjacent anatomical location was assessed, and part of the ilsilateral facet joint and the lamina was removed by a diamond burr. In regard to the range of decompression, depending on the severity of lesions, the METRx tube was moved up and down and the direction of the decompression was performed while adjusting the tube. The ligamentum flavum was removed and the nerve root and dura were assessed. If decompression of the contralateral side was required, then the retraction equipment was moved to the angle similar to the angle of the lamina, dis- section was initiated from the base of the spinous process and the inferior portion of the lamina at the contralateral side, and the dissection was continued to the lateral assess of the contralateral side. The contralateral ligamentum flavum was carefully removed using a punch, and afterward, the nerve root of the contralateral side could be assessed. In order to perform lumbar interbody fusion, the posterolateral annulus was removed and distraction of the disc space was performed by applying an interbody shaver, and the endplate was sufficiently removed using a curette. In most procedures, retraction of the dura and the nerve root was not required.

For interbody fusion, the contralateral side and the front area were adequately filled using bone fragments obtained during the operation and allobone in all cases. The capston cage was also filled, and it was inserted as anteriorly as possible. Afterward, in the same incision area, percutaneous pedicle screw fixation was performed, and rods were inserted and connected under $\mathrm{C}$-arm guidance. The identical procedures were performed on the opposite side.

\section{Clinical and radiographic evaluation}

For clinical evaluation, the visual analogue scale (VAS) score prior to operation and the Oswestry Disability Index (ODI) 2 weeks and 6 months after operation was analyzed. At the final follow-up, the clinical results were classified according to the Kirkaldy-Willis score. In addition, the operation time, the volume of intraoperative blood loss, the hospitalization period after operation and time until ambulation after the operation were analyzed.

For radiological evaluation, the disc space height, the segmental lumbar lordotic angle and the whole lumbar lordotic angle prior to surgery, 2 weeks after surgery, 6 months after surgery and at the final follow-up were analyzed. First, the disc space height was measured by the distance connecting the line drawn on the upper and lower endplates of the fusion segment and the center of the superior and inferior endplates. For the segmental lumbar lordotic angle, the angle formed by the upper and lower endplates of the fusion segments was measured. For the whole lumbar lordoric angle, the angle formed by the upper endplate of the L1 verterbral body and the upper endplate of the S1 verterbral body was measured.

For the evaluation of interbody fusion, at the final followup observation, the fusion rate was analyzed by applying the anterior fusion grade described by Bridwell et al. [5]. 
Independent sample t-tests were performed using the SPSS ver. 12.0 (SPSS Inc., Chicago, IL, USA) for testing the statistical significance of the radiological results prior to operation and at the final follow-up.

\section{Results}

\section{Clinical results}

In regard to the results of clinical evaluation, the VAS score of back pain was reduced from an average of 6.7 points prior to operation to an average of 1.8 points after operation. The ODI was decreased from an average of 36.5 points prior to operation to an average of 12.8 points after operation. In regard to the clinical results as assessed using the Kirkaldy-Willis score, 52 cases $(92.9 \%)$ showed better than good results (Table 1). In addition, based on the surgi- cal anesthesia records, the operation time was on average 144.2 minutes, and the volume of blood loss during operation was on average $410.6 \mathrm{ml}$. Based on the hospitalization records, the postsurgical hospitalization period was on average 14.6 days, and the time to postsurgical ambulation was on average 1.4 days.

\section{Radiological results}

In regard to the radiological evaluation, the disc space height was increased from $8.4 \pm 2.72 \mathrm{~mm}$ prior to operation to $12.2 \pm 1.91 \mathrm{~mm}$ two weeks after surgery, and this was $11.4 \pm 1.84 \mathrm{~mm}$ at the final follow-up $(p=0.002)$. The segmental lumbar lordotic angle was increased from 14.7 $\pm 9.42^{\circ}$ prior to operation to $18.5 \pm 6.52^{\circ} 2$ weeks after surgery, and this was $15.9 \pm 7.81^{\circ}$ at the final follow-up ( $p$ $=0.062)$. The whole lumbar lordotic angle was increased

Table 1. Clinical evaluation

\begin{tabular}{llcccc}
\hline \hline & & Pre op & Post op (2 wk) & Post op (6 mo) & Last F/U \\
\hline VAS & & 6.7 & 2.6 & 2.4 & 1.8 \\
ODI & 36.5 & 18.1 & 15.4 & 12.8 \\
Kirkaldy-Willis score & Excellent & & & $40(71)$ \\
& Good & & & $12(12)$ \\
& Fair & & & $2(3)$ \\
& Poor & & & $2(2)$ \\
& & & & 2 \\
& & & & \\
\end{tabular}

Values are presented as number (\%).

Pre op: Pre operation, Post op: Post operation, F/U: Follow up, VAS: Visual analogue scale, ODI: Oswestry Disability Index.
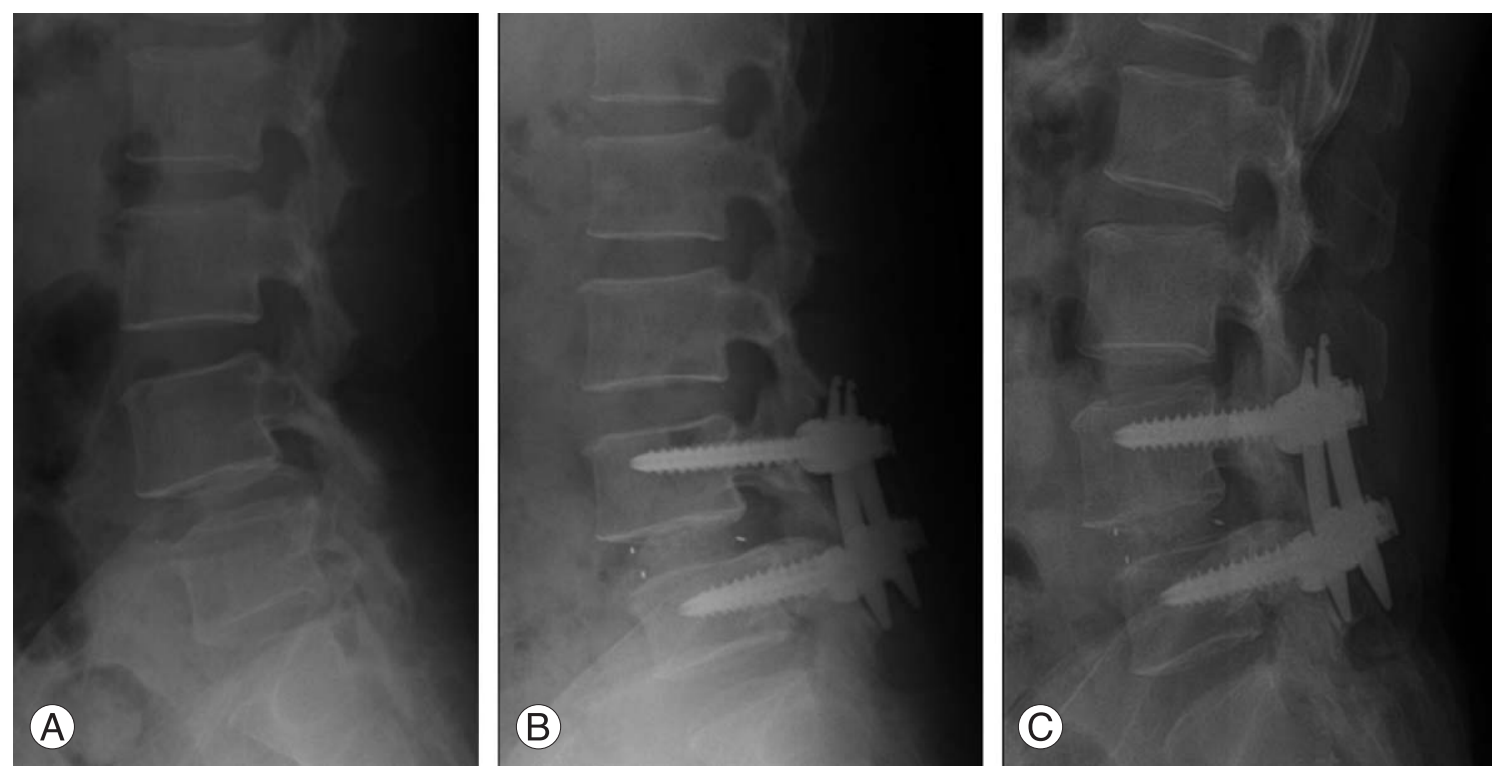

Fig. 1. The preoperative (A), 2 weeks postoperative (B), and last follow up (postoperative year, 3 yr) (C) lateral radiographs obtained in a 54-year-old man who underwent minimally invasive transforaminal lumbar interbody fusion. The last follow up radiographs demonstrate osseous union and improved lumbar lordosis. 
Table 2. Birdwell's anterial fusion grades

\begin{tabular}{llc}
\hline \hline Grade I & Fusion with remodelling and trabeculae & $41(73.2)$ \\
Grade II & Graft intact, not fully remodelled, no radiolucencies & $13(23.2)$ \\
Grade III & Graft intact, but a definite lucency & $1(1.8)$ \\
Grade IV & Definitely not fused, collapse & $1(1.8)$ \\
\hline
\end{tabular}

Values are presented as number $(\%)$.
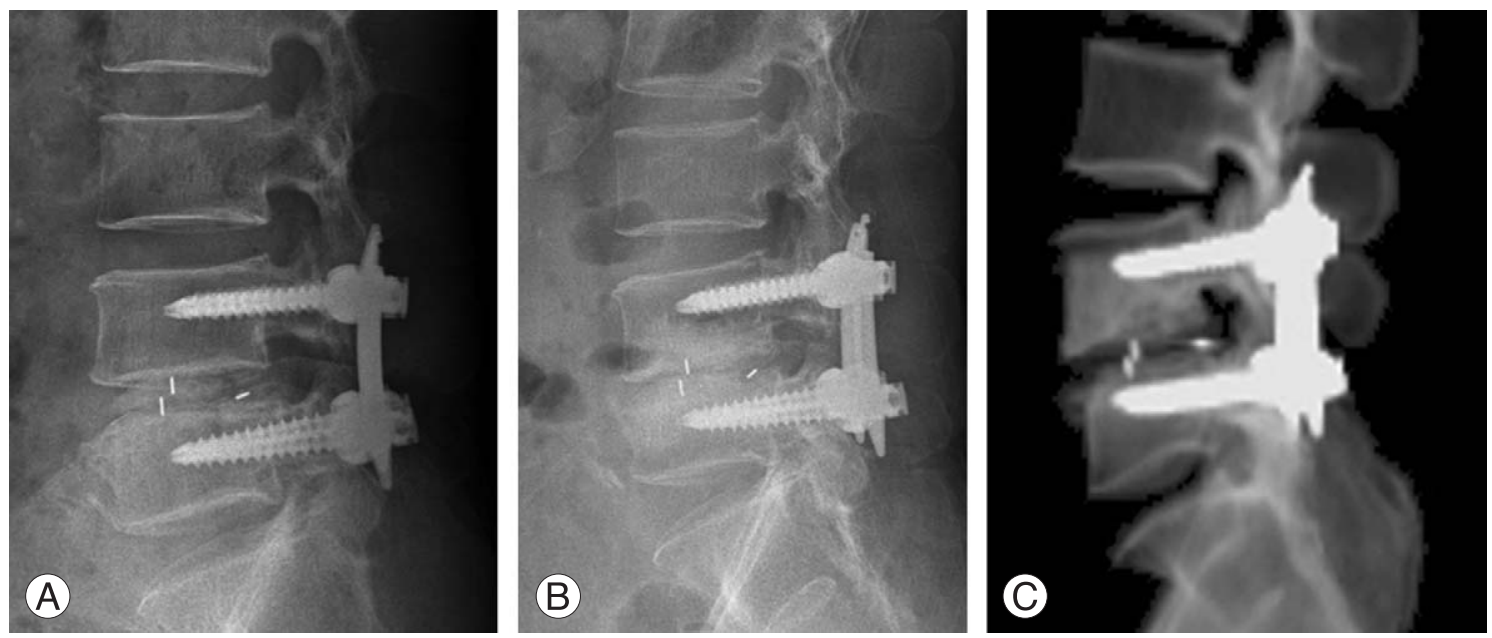

Fig. 2. The postperative radiograph showed a satisfactory cage position (A). Three months after surgery, the cage sank into vertebral body (B). At the 6 mo follow up, the sagittal computed tomography showed that the position of the cage had not changed and patient had moderate back pain, but no pathologic symptoms (C).

from $28.4 \pm 12.12^{\circ}$ prior to operation to $38.4 \pm 9.12^{\circ}$ two weeks after operation, and this was $35.8 \pm 11.82^{\circ}$ at the final follow up ( $p=0.001$ ) (Fig. 1). At the final follow-up, concerning the Birdwell's anterior fusion grade, there were 41 cases $(73.2 \%)$ and 13 cases $(23.2 \%)$ of grade I and grade II, respectively. On the dynamic radiographs, 2 cases showed unstable findings and this was determined to be non-union (Table 2, Fig. 2). As complications, the subsidence of cages developed in some patients, but this did not become a clinical problem.

\section{Discussion}

For the back pain and radiating pain symptoms caused by lumbar spinal degenerative diseases, surgical treatments can be considered if the cases do not improve despite of nonsurgical treatments. Traditional posterior lumbar interbody fusion may cause an increase of back pain due to resection in a wide area and resection of soft tissues, as well as a delay of recovery. Kawaguchi et al. [6] have reported on the damage of spinal muscles caused by the retractor blade during operation, and the marker of muscle injury (serum creatinine phosphokinase MM isoenzyme) values were shown to be proportional to the retraction pressure and the duration of retraction. To compensate for such shortcomings, numerous minimally invasive surgical techniques that minimize muscle injuries and soft tissues injuries during surgery have recently been introduced. Particularly, by the application of the metrix tube, the blood-loss volume during surgery is reduced and the denervation and contraction of the muscles of the adjacent soft tissues are prevented, and thus this has the advantages of reducing the postsurgical back pain and shortening the hospital stay.

Several investigators have reported that by performing transforaminal lumbar interbody fusion, decompression and bony fusion comparable to the previous anterior or posterial lumbar interbody fusion could be obtained [7-9]. We also performed follow-up observation of the clinical and radiological results and the procedure's effectiveness was examined. In regard to the clinical results, Potter et al. [10] have reported that in 100 patients with a minimum 2 years follow-up observation, satisfactory results were obtained in $80 \%$ of the patients. Foley et al. [3] have reported that the ODI was reduced from 55 prior to operation to 11 after operation. We also applied the VAS score, the ODI and the Kirkaldy-Willis score. The VAS score was reduced from an 
average of 6.7 points prior to operation to an average of 1.8 points after operation. The ODI was reduced from 36.5 points prior to operation to an average of 12.8 points after operation. For the clinical results assessed by the KirkaldyWillis score, 52 cases (92.9\%) in our study showed similar results compared to Foley et al.'s results [3]. In addition, the anatomical differences of patients, the experience of surgeons and several other factors may exert effects on the operation time or the blood-loss volume during operation. Relton and Hall [11] analyzed the factors that may exert effects of the blood-loss volume during surgery, and they reported that these factors are general anesthesia during surgery, the pattern of mechanical ventilation, the tension of the diaphragm and the anterior abdominal wall muscles, the patient's posture during surgery and other factors. Hence, in our report, the results of the operation time, the blood-loss volume during surgery, the time till postsurgical ambulation and the hospitalization period have limitations since they were based only on the medical records, and we think that that more prospective studies on this are required. In regard to the radiological results, according to Hackenberg et al. [12], the success rate of bony fusion of transforaminal lumbar interbody fusion has been reported to be approximately $86 \%$, and this fusion rate was comparable to our rate. Potter et al. [10] have reported that for obtaining firm interbody fusion, exposure of more than $30 \%$ of the endplate of the interbody is required, and clinically, by using the unilateral transforaminal approach, an average of $69 \%$ of the disc volume (56\% of the endplate) could be removed. It has been reported that even by the transforaminal approach, the anterior interbody could be firmly fused and the lumbar lordotic angle could be increased. In addition, by preserving the posterior ligament complex and the contralateral facet joint, it has advantages that iatrogenic instability could be less induced and so even biomechanically the fusion rate could be increased. In our cases the disc space height of the fusion segments was increased from $8.4 \pm 2.72 \mathrm{~mm}$ prior to operation to $12.2 \pm 1.91 \mathrm{~mm}$ after operation and it was $11.4 \pm$ $1.84 \mathrm{~mm}$ at the final follow-up observation. The lumbar lordotic angle was increased from $28.4 \pm 12.12^{\circ}$ prior to operation to $38.4 \pm 9.12^{\circ}$ after operation, and at the final follow-up observation, it was $35.8 \pm 11.82^{\circ}$ and maintained well.

However, the lordotic angle of the fusion segments was increased from $14.7 \pm 9.42^{\circ}$ prior to operation to $18.5 \pm$ $6.52^{\circ}$ after operation, and it was decreased to $15.9 \pm 7.81^{\circ}$ at the final follow-up. Nonetheless, the difference was not statistically significant. We can speculate that this is due to that during the endplate preparation for the insertion, loss of bony cartilage occurred and so subsidence of the cages developed, or the capstone cage (Medtronic) was not in a wedge shape and so the lordotic angle of the fusion segments could not be maintained well, in part. To overcome this, Kim et al. [13] have reported that to maintain the lordotic angle of the fusion segments, the loss of bony cartilages should not occur during the endplate preparation, and it is recommended to graft local bones to the anterior area of the cages and subsequently to insert the possible cages in the anterior area, and the insertion of wedge shaped cages may be required. In addition, as compared with the insertion of two cages, the insertion of one cage may be less mechanically fused or maintenance of the lordotic angle may be difficult. Nonetheless, according to the study reported by Humphreys et al. [14] the use of a single cage may not cause problems. However, as mentioned previously, minimally invasive transforaminal fusion has several advantages for the clinical and radiological results as compared with traditional fusions, yet the former still has some limitations. Since minimally invasive transforaminal fusion has to be performed within a small tubular retractor and under a microscope, it has shortcomings that manipulation is not easy, and a long time is required to acquire the proper surgical techniques. Foley et al. [3] have also reported that during the initial period, the mean operation time was approximately 240 minutes, and in our cases, the average operation time was 220 minutes during the initial period. Yet for the single level fusion cases, the mean operation time was an average of 144 minutes and then it substantially decreased. Another limitation is that the complications that occur during an operation may be difficult to treat in many cases. Particularly, for cases with the damaged dura, general primary repair was difficult in many cases. Nonetheless, the size of the damage to the dura was small, and it was resolved by spraying cerebrospinal fluid (CSF) sealant, etc. It was sutured in some cases, but complications caused by CSF leakage did not develop, and after operation the positive pressure suction drain was maintained, and from 2 days after operation, the drain was removed and walking was initiated. In addition, during the endplate preparation procedure, the subsidence of cages caused by the loss of bony cartilage did not cause clinical symptoms, and so only comprehensive follow-up observation was performed. Although this study reports on only transforaminal lumbal interbody fusion limited to a single level, decompression could be per- 
formed on two or three levels. Min and Lee [15] have reported that if the disc space is substantially narrowed, or in the cases higher than the Meyerding grade II, it may be preferable to perform surgery by the bilateral approach, and it is considered that in surgeries for most other degenerative lumbar diseases, special limitations may not be required. In addition, by minimizing the soft tissue injuries, this could reduce the recovery period after the operation as well as the pain. It is considered that if surgeons become familiar with the techniques, then these could become the techniques for mechanically maintaining the lumbar lordosis and high bone fusion rates can be obtained. The limitations of our study are that the surgery was performed from 2005, but the follow-up period was not sufficient, and so longer-term follow and comparison with the previous methods are required, together with larger scale prospective studies.

\section{Conclusions}

In patients who undergo minimally invasive transforaminal lumbar interbody single level fusion, there is less soft tissue injuries and the recovery from back pain after the operation is better and the time until ambulation can be shortened. Radiologically, the bone fusion rate was good and the lumbar lordotic angle was well maintained. We think that this minimally invasive surgical method is effective for treating degenerative lumbar diseases.

\section{REFERENCES}

1. Thomsen K, Christensen FB, Eiskjaer SP, Hansen ES, Fruensgaard S, Bünger CE. 1997 Volvo Award winner in clinical studies. The effect of pedicle screw instrumentation on functional outcome and fusion rates in posterolateral lumbar spinal fusion: a prospective, randomized clinical study. Spine (Phila Pa 1976) 1997;22:2813-22.

2. Steffee AD, Sitkowski DJ. Posterior lumbar interbody fusion and plates. Clin Orthop Relat Res 1988;227:99-102.

3. Foley KT, Holly LT, Schwender JD. Minimally invasive lumbar fusion. Spine (Phila Pa 1976) 2003;28(15 Suppl): S26-35.

4. Schwender JD, Holly LT, Rouben DP, Foley KT. Minimally invasive transforaminal lumbar interbody fusion (TLIF): technical feasibility and initial results. J Spinal Disord Tech 2005;18 Suppl:S1-6.
5. Bridwell KH, Lenke LG, McEnery KW, Baldus C, Blanke $\mathrm{K}$. Anterior fresh frozen structural allografts in the thoracic and lumbar spine. Do they work if combined with posterior fusion and instrumentation in adult patients with kyphosis or anterior column defects? Spine (Phila Pa 1976) 1995;20: 1410-8.

6. Kawaguchi Y, Matsui H, Tsuji H. Back muscle injury after posterior lumbar spine surgery: a histologic and enzymatic analysis. Spine (Phila Pa 1976) 1996;21:941-4.

7. Park P, Foley KT. Minimally invasive transforaminal lumbar interbody fusion with reduction of spondylolisthesis: technique and outcomes after a minimum of 2 years' follow-up. Neurosurg Focus 2008;25:E16.

8. Yan DL, Pei FX, Li J, Soo CL. Comparative study of PILF and TLIF treatment in adult degenerative spondylolisthesis. Eur Spine J 2008;17:1311-6.

9. Park Y, Ha JW. Comparison of one-level posterior lumbar interbody fusion performed with a minimally invasive approach or a traditional open approach. Spine (Phila Pa 1976) 2007;32:537-43.

10. Potter BK, Freedman BA, Verwiebe EG, Hall JM, Polly DW Jr, Kuklo TR. Transforaminal lumbar interbody fusion: clinical and radiographic results and complications in 100 consecutive patients. J Spinal Disord Tech 2005; 18:337-46.

11. Relton JE, Hall JE. An operation frame for spinal fusion: a new apparatus designed to reduce haemorrhage during operation. J Bone Joint Surg Br 1967;49:327-32.

12. Hackenberg L, Halm H, Bullmann V, Vieth V, Schneider M, Liljenqvist U. Transforaminal lumbar interbody fusion: a safe technique with satisfactory three to five year results. Eur Spine J 2005;14:551-8.

13. Kim SB, Jeon TS, Heo YM, et al. Radiographic results of single level transforaminal lumbar interbody fusion in degenerative lumbar spine disease: focusing on changes of segmental lordosis in fusion segment. Clin Orthop Surg 2009;1:207-13.

14. Humphreys SC, Hodges SD, Patwardhan AG, Eck JC, Murphy RB, Covington LA. Comparison of posterior and transforaminal approaches to lumbar interbody fusion. Spine (Phila Pa 1976) 2001;26:567-71.

15. Min SH, Lee DH. Minimally invasive transforaminal lumbar interbody fusion in patients with low grade spondylolisthesis: comparison of the unilateral and bilateral approaches. J Korean Orthop Assoc 2009;44:429-35. 